\section{Diagnosis of Hyperprogressive Disease in Patients Treated with Checkpoint Inhibitors Using ${ }^{18}$ F-FDG PET/CT}

TO THE EDITOR: A recent ahead-of-print publication by Castello et al. (1) provides fascinating insights on the potential prognostic role of ${ }^{18} \mathrm{~F}$-FDG PET/CT in patients with non-small cell lung cancer treated with immune checkpoint inhibitors (ICIs). The authors unraveled the prognostic value of mainstream quantitative imaging biomarkers that can be derived from ${ }^{18} \mathrm{~F}$-FDG PET using most clinical workstations. They confirmed that baseline tumor burden, baseline total lesion glycolysis on PET scans, and derived neutrophil-to-lymphocyte ratio (neutrophils/leukocytes minus neutrophils) were associated with overall survival in cancer patients treated with ICIs (2).

In this timely and comprehensive work written by experts in this field, the authors mined the data contained in baseline PET images from 50 non-small cell lung cancer patients treated with ICIs. They evaluated the association between overall survival and 8 candidate biomarkers, including imaging $(n=4)$ and biologic $(n=4)$ variables. They used a previously published composite criterion to diagnose hyperprogression (3), which can be simplified as patients with fast tumor growth during the first 2 mo of treatment. The important point is that these fast, progressive patients might have already been progressing rapidly before the initiation of ICIs. This fast progression during the first 2 mo of treatment was observed in 1 of 3 patients $(30 \%, n=14 / 46)$ treated with ICIs (1) and was more frequent in patients with higher baseline tumor burden, a higher number of metastatic sites, and proinflammatory parameters (pretreatment derived neutrophil-tolymphocyte ratio and platelet counts). Such research should be actively pursued since it is of tremendous significance.

In this new era of immune-oncology, the treatment paradigm is shifting toward restoring tumor elimination by the immune system, hence the emergence of novel patterns of response (4) and progression, such as pseudoprogression (5) and hyperprogression (6). Although the medical community has gained experience in the management of pseudoprogression (5), the current wait-and-see strategy proposed to take into account delayed radiographic shrinkage is challenged by hyperprogression. Hyperprogression is indeed an atypical flair-up of tumor growth kinetics linked to premature death (6-8) due to a harmful effect of immunotherapy (6). Hyperprogression is a clinical phenomenon that might be underdiagnosed since it is a new concept that has only recently emerged. ICIs might harm $4 \%$ (9) to $29 \%$ (7) of patients with solid tumors through an accelerated progression profile leading to premature death. The underlying mechanism is an area of active investigation. Reported risk factors are a higher age ( 6 ) and the presence of MDM2/4 family amplification or estimated glomerular filtration rate aberrations (9).

The frequency of hyperprogression in patients with non-small cell lung cancer treated with ICIs differs widely in the literature: it was $30 \%$ in the study of Castello et al. as compared with $8 \%$ (3/38) (9) and 14\% (56/406) (8) in other series. The current challenge

COPYRIGHT @ 2020 by the Society of Nuclear Medicine and Molecular Imaging. is that distinct definitions are proposed. The same term, hyperprogression, is now used to conceptualize 2 distinct pathophysiologic phenomenon: a fast progression that may not be due to ICIs (prognostic tool) or an accelerated tumor growth after the initiation of ICIs associated with premature death (predictive tool).

In one definition, hyperprogression defines a fast progression that may be independent of ICIs. This strategy considers tumor growth rate only after the initiation of ICIs $(1,3)$. This strategy is convenient since it requires only two response assessments. Nonetheless, this definition cannot demonstrate a causality effect: the fast progression profile cannot be attributed specifically to immunotherapy. Since cancers have exponential growth, patients with a high baseline tumor burden are, therefore, more likely to progress more quickly and to be called hyperprogressors if we only consider these two time points.

In another definition, hyperprogression defines an accelerated progression attributed to a harmful effect of immunotherapy $(6,8)$. This strategy considers a change between pretreatment tumor growth rate and on-treatment tumor growth rate and has demonstrated a low rate of hyperprogression using PET (10). This definition aims to identify predictive biomarkers associated with a dramatic surge in tumor growth due to immunotherapy. Such a definition presupposes medical imaging before, at the start of, and during ICIs; such imaging is often available in clinical practice in patients treated with ICIs as the second line of therapy or if there is a wait time from referral to first treatment. The median time reported in the literature is $6 \mathrm{wk}$ for first-line treatment of non-small cell lung cancer.

In conclusion, given the clinical and prognostic importance of hyperprogression, it is important to harmonize the criteria for its definition. These criteria could be a combination of clinical, radiologic (CT), and metabolic (PET) data. Beyond the definition, it is important to take into account and differentiate two distinct mechanisms-fast progression and accelerated progression-and harmonize how they are highlighted (through biomarkers, periodicity, or other means). Finally, it is necessary to harmonize the technical criteria for measuring target lesions, including whether new lesions are considered, and clinical data.

\section{REFERENCES}

1. Castello A, Rossi S, Toschi L, Mazziotti E, Lopci E. Hyper-progressive disease in patients with non-small cell lung cancer treated with checkpoint inhibitors: the role of ${ }^{18}$ F-FDG PET/CT. $J$ Nucl Med. December 20, 2019 [Epub ahead of print].

2. Seban RD, Mezquita L, Berenbaum A, et al. Baseline metabolic tumor burden on FDG PET/CT scans predicts outcome in advanced NSCLC patients treated with immune checkpoint inhibitors. Eur J Nucl Med Mol Imaging. 2020;47:1147-1157.

3. Lo Russo G, Moro M, Sommariva M, et al. Antibody-Fc/FcR Interaction on macrophages as a mechanism for hyperprogressive disease in non-small cell lung cancer subsequent to PD-1/PD-L1 blockade. Clin Cancer Res. 2019;25:989-999.

4. Borcoman E, Kanjanapan Y, Champiat S, et al. Novel patterns of response under immunotherapy. Ann Oncol. 2019;30:385-396.

5. Chiou VL, Burotto M. Pseudoprogression and immune-related response in solid tumors. J Clin Oncol. 2015;33:3541-3543.

6. Champiat S, Dercle L, Ammari S, et al. Hyperprogressive disease is a new pattern of progression in cancer patients treated by anti-PD-1/PD-L1. Clin Cancer Res. 2017;23:1920-1928.

7. Saâda-Bouzid E, Defaucheux C, Karabajakian A, et al. Hyperprogression during anti-PD-1/PD-L1 therapy in patients with recurrent and/or metastatic head and neck squamous cell carcinoma. Ann Oncol. 2017;28:1605-1611.

8. Ferrara R, Mezquita L, Texier M, et al. Hyperprogressive disease in patients with advanced non-small cell lung cancer treated with PD-1/PD-L1 inhibitors or with single-agent chemotherapy. JAMA Oncol. 2018;4:1543-1552. 
9. Kato S, Goodman A, Walavalkar V, Barkauskas DA, Sharabi A, Kurzrock R. Hyperprogressors after immunotherapy: analysis of genomic alterations associated with accelerated growth rate. Clin Cancer Res. 2017;23:4242-4250.

10. Dercle L, Seban R-D, Lazarovici J, et al. ${ }^{18}$ F-FDG PET and CT scans detect new imaging patterns of response and progression in patients with Hodgkin lymphoma treated by anti-programmed death 1 immune checkpoint inhibitor. J Nucl Med. 2018;59:15-24.

Romain-David Seban
Lawrence H. Schwartz
Gerald Bonardel
Laurent Dercle*
*Department of Radiology at Columbia
University Medical Center/NewYork-Presbyterian Hospital
168 th St.
New York, NY 10032
E-mail: ld2752@ cumc.columbia.edu

Published online Feb. 21, 2020.

DOI: $10.2967 /$ jnumed.120.242768

REPLY: We thank Seban et al. for their interest and their insightful comments on our study (1). We very much agree with them on the remarkable potential role of the quantitative parameters derived from ${ }^{18} \mathrm{~F}-\mathrm{FDG}$ PET/CT in predicting response to immune checkpoint inhibitors (ICIs). Furthermore, as has emerged from the latest publications, the combination of ICIs with circulating biomarkers such as neutrophil-to-lymphocyte ratio, derived neutrophil-to-lymphocyte ratio, circulating tumor cells, and cell-free DNA can provide complementary information and appears promising in predicting clinical outcomes.

However, we believe that some aspects require more thorough clarification. On the basis of the 2 time points (baseline and $8 \mathrm{wk}$ after ICI start) used in our study to define hyperprogressive disease (HPD) (1), Seban et al. affirm that patients might already have been progressing rapidly before the initiation of ICI. Indeed, most classifications define HPD by using tumor growth rate (TGR), which considers the tumor growth during ICI treatment in comparison with a reference period immediately before ICI. Nevertheless, this computation of TGR is not free from drawbacks and might underestimate the real number of patients experiencing HPD, primarily because the assessment of new lesions and nonmeasurable disease is not considered in the definition of TGR (whereas we know quite well that progressive disease often is driven by the appearance of new lesions or an increase in nontarget lesions) and secondarily because it can be difficult to reach a TGR doubling in tumors with a higher TGR before treatment. For instance, an increase from $60 \%$ before ICI to $80 \%$ during ICI treatment will not configure HPD on the basis of the above criteria, despite a significant absolute increase in tumor burden. In other words, using TGR might exclude HPD in tumors with a large tumor burden before ICI. Similarly, nonmeasurable lesions, for example, lymphangitis, bone metastases, and pleural or peritoneal effusions, might not be represented in the whole tumor burden based on pure morphologic criteria (RECIST). In this regard, we must not forget that a high number of metastatic sites can be as valid surrogate of tumor burden, as has emerged in previous studies (2). Along with the TGR clinical limits, there is also a logistical limitation: TGR computation requires a prior CT scan, which is sometimes difficult to retrieve; for example, a prior CT scan could not be retrieved in $30 \%$ of the cases in the study of Matos et al. (3). Therefore, in our criteria we also included time to treatment failure, which can be clinically useful when TGR cannot be evaluated.
Finally, Seban et al. highlight the high prevalence of HPD in our study, that is, $30 \%$, compared with other series. Besides the different criteria used in defining HPD, most other studies include all tumor types, whereas our cohort was limited to non-small cell lung cancer patients. When only this tumor type is considered, our results are quite consistent with those of other studies dealing with a similar patient cohort (2).

In the end, what comes out of our study is that we were able to identify a subgroup of patients with a worse outcome during ICI therapy, and this ability alone is relevant evidence independently of whether it resulted from the treatment itself or the intrinsic behavior of the tumor. In our opinion, distinction between fast and accelerated progression is still premature and is a purely semantic license so far, because methods proposed for HPD have their own limitations. Therefore, a universally accepted consensus on how to define and measure HPD is necessary, and that need for a universally accepted consensus is in line with our conclusions and those derived by Seban et al. in their letter to the editor.

\section{DISCLOSURE}

Egesta Lopci was awarded an individual grant, 18923, by AIRC (Associazione Italiana per la Ricerca sul Cancro). Angelo Castello is supported by fellowships related to the same AIRC grant. No other potential conflict of interest relevant to this article was reported.

\section{REFERENCES}

1. Castello A, Rossi S, Toschi L, Mazziotti E, Lopci E. Hyper-progressive disease in patients with non-small cell lung cancer treated with checkpointi: the role of ${ }^{18}$ F-FDG PET/CT. J Nucl Med. December 20, 2019 [Epub ahead of print].

2. Kim CG, Kim KH, Pyo KH, et al. Hyperprogressive disease during PD-1/PD-L1 blockade in patients with non-small-cell lung cancer. Ann Oncol. 2019;30:11041113.

3. Matos I, Martin-Liberal J, García-Ruiz A, et al. Capturing hyperprogressive disease with immune-checkpoint inhibitors using RECIST 1.1 criteria. Clin Cancer Res. 2020;26:1846-1855.

Angelo Castello
Egesta Lopci*
*Humanitas Clinical and Research Hospital-IRCCS
Via Manzoni 56, 20089
Rozzano, Milan, Italy
E-mail: egesta.lopci@gmail.com

Published online Mar. 13, 2020.

DOI: 10.2967/jnumed.120.243444

\section{SUV $_{\text {max-V }}$ for Assessing Treatment Response in ${ }^{18}$ F-FDG PET Imaging of Patient-Derived Tumor Xenografts Involving Triple-Negative Breast Cancer}

TO THE EDITOR: In the preclinical arm of a coclinical trial, Savaikar et al. recently optimized ${ }^{18}$ F-FDG PET imaging biomarkers of response to a combined docetaxel and carboplatin therapy in patient-derived tumor xenografts involving triple-negative breast cancer (1). Twenty-one necrotic-core-phenotype tumors and 